\title{
Precapillary systemic-pulmonary anastomoses
}

\author{
MAR GARET T UR NER-WAR WICK \\ From the Institute of Diseases of the Chest, London, S.W.3
}

In normal lungs the bronchial arteries communicate with the pulmonary arteries through the capillary bed surrounding the respiratory bronchioles (Miller, 1947). Whether precapillary communications exist in normal lungs is open to question, but their development under abnormal circumstances is generally agreed. Liebow, Hales, and Bloomer (1959) have summarized the two main circumstances under which they have been found: $(a)$ obstruction and diminished flow in the pulmonary arteries, and (b) formation of new tissue in the lung.

Many different anatomical forms of systemicpulmonary anastomoses can be found in the lung, but hitherto only a few of these have been described in detail owing to the limitations of the techniques used. Cast preparations have been of great value in identifying the larger communications, but this method has two disadvantages. Firstly, histological verification of anastomoses is impossible, and close apposition of minute vessels may be indistinguishable from their actual continuity. Secondly, the histological study of vessel wall structure and the surrounding tissue is denied. Angiographic studies of whole lungs have been of limited value because systemicpulmonary anastomoses cannot be distinguished from superimposition of non-communicating vessels.

In the present work a microradiographic technique has been developed to study the finer branches of the bronchial arteries and their sites of communication with the pulmonary vascular bed. The findings have been verified by serial sections.

\section{THE MORPHOLOGY OF PREVIOUSLY DESCRIBED SYSTEMIC-PULMONARY ANASTOMOSES}

NORMAL LUNGS Verloop (1948) studied the histology of normal lungs and described two forms of anastomoses. 'Deep' communications were described in the neighbourhood of small bronchi, where the thick-walled bronchial artery gave rise to a thin-walled lateral 'connecting' vessel which ran distally, lying in convolutions on the wall of the bronchus, and ended in ramifications of the pulmonary artery. The 'superficial' communications were not described in detail but were believed to occur between the bronchial arteries lying in the interlobular septa and branches of the pulmonary artery. Harris and Heath (1962), using histological methods, were unable to confirm Verloop's finding of 'deep' anastomoses, and other workers using injection techniques have also failed to demonstrate these anastomoses in normal lung (Cudkowicz and Armstrong, 1951; Weibel, 1959).

ABNORMAL LUNGS (CONGENITAL HEART DISEASE AND ACQUIRED LUNG DISEASE)

Pulmonary stenosis Hales and Liebow (1948) described two forms of anastomoses in lungs from cases of pulmonary stenosis. One type was found close to the hilum, where a major bronchial artery branch connected directly with the accompanying segmental axial pulmonary artery in an end-to-side manner. More peripheral end-to-side communications were also reported, the bronchial artery joining a lobular branch of the pulmonary artery.

Pulmonary artery occlusion In experimental pulmonary artery ligation in dogs, peripheral anastomoses have been found between bronchial artery branches and lobular pulmonary arteries similar in form to those seen in congenital pulmonary stenosis (Liebow, Hales, Bloomer, Harrison, and Lindskog, 1950 ; Cockett and Vass, 1951).

At sites of thrombotic obstruction of the pulmonary artery Cudkowicz and Armstrong (1953a) have described an entirely different form of anastomosis. Vasa vasorum (from the systemic circulation) were found penetrating the pulmonary arterial wall to establish continuity with its lumen. They believed that acquired bronchopulmonary anastomoses seen in many lung diseases were formed in this way.

Bronchiectasis Anastomoses of two types have been described. A proximal type was found at the level of the third to sixth generation bronchus where the axial pulmonary artery joined the accompanying bronchial artery through a distinct 
connecting vessel which linked the parallel arteries like the strut of a ladder. More distally, anastomoses have been found in relation to bronchiectatic sacs (Liebow, Hales, and Lindskog, 1949 ; Cockett and Vass, 1951).

Many other workers using injection techniques have demonstrated the presence of bronchopulmonary connexions in abnormal lungs by observing overflow of medium from the systemic to the pulmonary circulation, but the morphology of these anastomoses was not usually described in detail.

\section{TERMINOLOGY}

In this paper the term 'systemic arterial supply' to the lungs refers to all vessels supplying the lung arising directly or indirectly from the aorta, and includes both the bronchial arteries reaching the lung on the posterior wall of the main bronchi at the hilum, and the accessory arteries reaching the lung through the hilum and the pulmonary ligament.

'Subpleural systemic-pulmonary anastomoses' means the precapillary connexions between the systemic and pulmonary arteries lying immediately under the visceral pleura. The systemic component may arise either from the bronchial arteries which reach the pleura along the interlobular septa or from the accessory arteries reaching the pleura through the pulmonary ligament.

'Intrapulmonary systemic-pulmonary anastomoses' means the precapillary connexions between the systemic and pulmonary arteries lying within the substance of the lung.

\section{MATERIAL AND METHODS}

A total of 118 lungs have been studied from 64 patients with various lung and cardiac diseases.

The systemic arteries to the lung were injected with a suspension of barium sulphate as $\mathbf{8 0} \%$ 'Micropaque' (Demancey) in 15\% gelatin. After fixation in 10\% formalin, sagittal slices of individual lung lobes were cut $0.5 \mathrm{~cm}$. thick and studied using a microradiographic technique described previously (TurnerWarwick, 1961). Using this method, vessels of $30 \mu$ and greater were filled regularly, but capillaries remained uninjected.

In 15 normal lungs, no filling of the pulmonary arterial bed occurred after injection of the bronchial arteries, indicating that no precapillary systemicpulmonary anastomoses were present in healthy lungs. In abnormal lungs anastomoses were found frequently, and the different morphological types will be described.

\section{RESULTS}

CONGENITAL PULMONARY STENOSIS Nine lungs have been studied from patients with Fallot's tetralogy with severe pulmonary stenosis. Systemicpulmonary connexions were demonstrated in all cases, but the number found in individual lungs varied considerably. This variability has been noted before by Hales and Liebow (1948).

The largest anastomoses were found between branches of the systemic arteries lying on the visceral mediastinal pleura and peripheral pulmonary arteries. The majority of these pleural branches were derived from true bronchial arteries which entered the lung with the main bronchi at the hilum and reached the mediastinal surface through the interlobular septa. Thereafter these branches radiated over the mediastinal surface lying immediately under the visceral pleura. From this surface network lateral branches, 200-400 $\mu$ in diameter, were found which communicated end to end with underlying branches of the pulmonary artery. The pulmonary components of these anastomoses were larger than the other branches of the pulmonary artery in the immediate vicinity ; this feature simplified the identification of these 'isolated' subpleural systemic-pulmonary anastomoses (Fig. 1a).

Anastomoses of identical form were also found between accessory systemic arteries which reached the lung through the pulmonary ligament. For this reason the greatest numbers of subpleural systemic-pulmonary anastomoses were found over the mediastinal aspect of the lower lobes, in the region where these accessory vessels entered the lung. The majority of communications were found within $5 \mathrm{~cm}$. of the hilum, but occasional ones were identified over the entire mediastinal surface. Although the largest anastomoses were about $400 \mu$ in diameter, innumerable smaller ones were dissected. In the absence of pleural adhesions,

FIG. 1 (opposite). Fallot's tetralogy. The systemic arteries only have been injected and the pulmonary arterial bed $\mathrm{N}$ filled through systemic-pulmonary anastomoses. (a) Tortuous systemic arterial branches under the visceral $\mathbb{N}$ mediastinal pleura of the lung; one branch is shown forming an 'isolated' end-to-end anastomosis with a peripheral branch of the pulmonary artery. (b) A bronchial artery branch is shown leaving its accompanying pulmonary artery, running outwards into the surrounding $\mathbb{\infty}$ lung tissue to communicate with a distant pulmonary? artery branch. (c) A bronchial artery branch is shown communicating end-to-end with a lateral branch of the $\frac{\vec{\Phi}}{\vec{\Phi}}$ accompanying pulmonary artery. Serial section confirmed that the vessel changed from systemic to pulmonary type at the point marked in the diagram (level 2). 


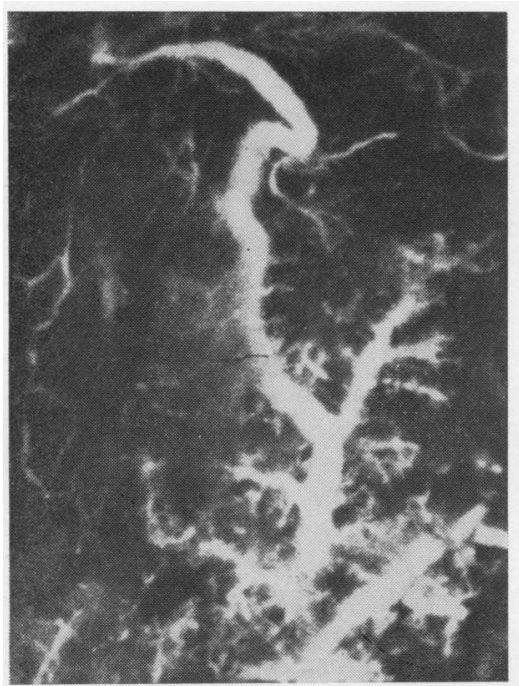

FIG. 1a

Pulmonary artery
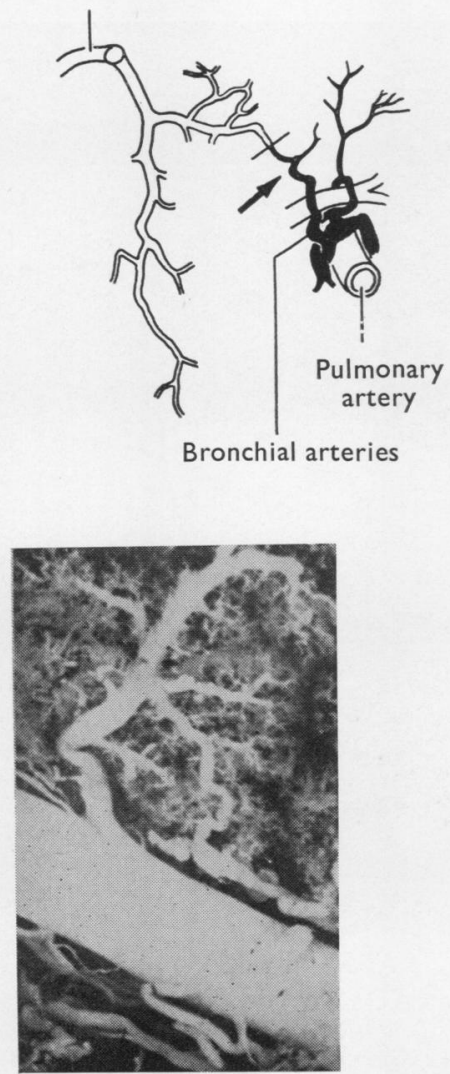

FIG. 1c
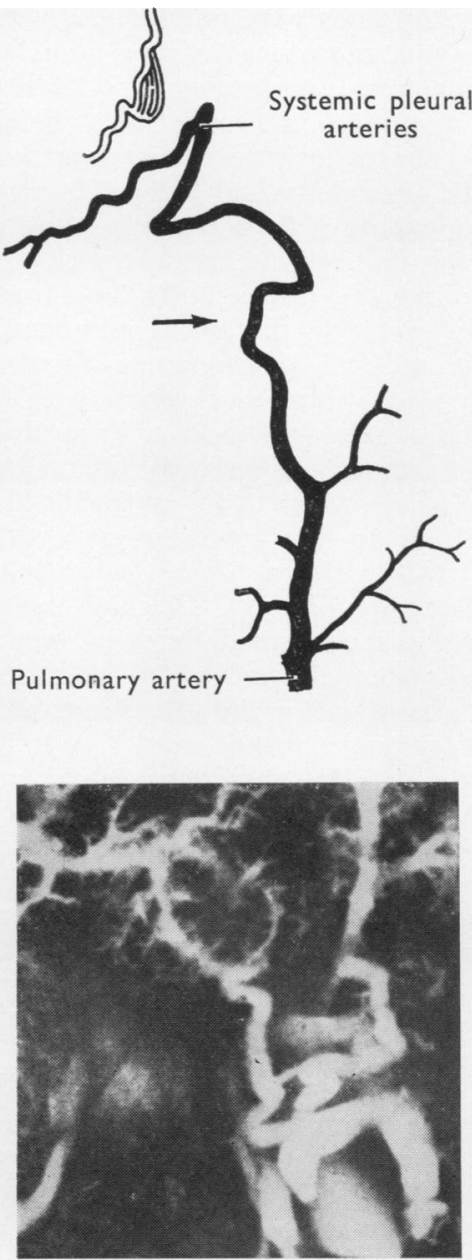

FIG. $1 \mathrm{~b}$

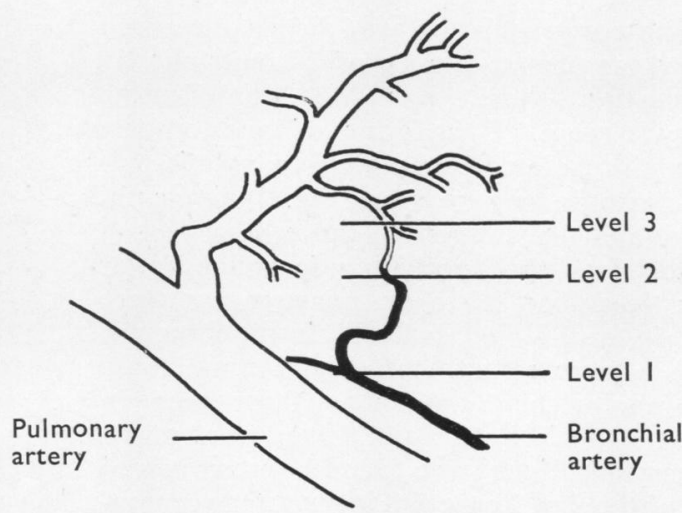


pleural anastomoses were not found on the costal or diaphragmatic surfaces of the lung, which in man do not receive a systemic arterial supply.

Within the lung numerous anastomoses were found, and the majority of these occurred within $5 \mathrm{~cm}$. of the hilar pleural reflection. The pulmonary vessel components of these connexions were lateral branches arising from the first to fourth generation of the pulmonary arteries. No communications were found entering the axial pulmonary artery directly, at any level, although many large tortuous bronchial artery branches were seen running parallel and closely applied to it. From these main bronchial arteries, numerous lateral systemic branches arose which ran outwards into the adjacent lung to join end to end with small branches from adjacent and distant pulmonary arteries (Fig. 1b; Fig. 1c). The end-to-end mode of junction was confirmed by serial section: the thin-walled systemic vessel in Fig. 1c left its parent bronchial artery and ran into the surrounding lung and as a systemic vessel gave rise to no side branches (level 1). In subsequent sections arteriolar branches arose from this vessel and reached the alveolar walls, thus defining the vessel as a pulmonary arteriole (level 2). In later sections still, it could be seen joining a definitive small muscular pulmonary artery with characteristic wall structure (level 3). In this way the end-to-end mode of anastomosis between the bronchial and pulmonary vessels was ascertained.

There was no evidence of intrapulmonary occlusion by thrombus in the region of these anastomoses and no evidence that the formation of these anastomoses was related to acquired intrapulmonary vascular obstruction.

CONGENITAL HEART DISEASE WITH PULMONARY HYPERTENSION Two lungs were studied from a patient with cor triatriatum who had a measured pulmonary vascular resistance of 20 units shortly before death; there was no complicating pulmonary disease. Two forms of anastomoses were found. Isolated subpleural systemic-pulmonary anastomoses were seen (Fig. 2) similar in form, size, and distribution to those described in Fallot's tetralogy, but occurring in much fewer numbers: less than 10 such anastomoses were identified in each lung. Isolated intrapulmonary anastomoses were also found (Fig. 3), and these were morphologically similar to those seen in pulmonary stenosis but, again, fewer in number. These communications were found between lateral branches of the first four pulmonary artery generations. Occasional more peripheral communications were probably demonstrated (Fig. 3), but as it was not possible to identify these vessels with certainty $\frac{\bar{\sigma}}{\overline{0}}$ on serial section, the vascular continuity between $\widetilde{\Phi}$ the systemic and pulmonary circulations in the lung periphery was not proved.

Two lungs were examined from a patient with $\overrightarrow{0}$ Eisenmenger's syndrome and ventricular septal $\overrightarrow{-}$ defect: in these a more complex situation was ${ }_{\sigma}$ found owing to the superimposition of acquired $\stackrel{F}{F}$ lung disease. 'Isolated' subpleural systemic-pul- $\vec{x}$ monary anastomoses were found similar to those $\vec{\infty}$ seen in cor triatriatum (Fig. 2), but within the lung $\omega$ no end-to-end anastomoses were seen. 'Angio- N matoid' lesions described previously by Heath and ${ }_{0}^{\mathrm{O}}$ Edwards (1958) were extremely numerous in this case. 'Angiomatoid' lesions occur in advanced $\vec{\infty}$ pulmonary hypertension: small muscular pul- $\mathbb{D}$ monary arteries showing intimal fibrosis give rise $\underset{\mathbb{D}}{\mathbb{D}}$ to thin-walled vascular formations which occupy 3 the lumen of the pulmonary vessel and extend out- $\frac{\mathbb{Q}}{-}$ wards to surround it. The relation of these $\vec{\oplus}$ 'angiomatoid' lesions to the systemic vascular bed $\stackrel{\oplus}{\oplus}$

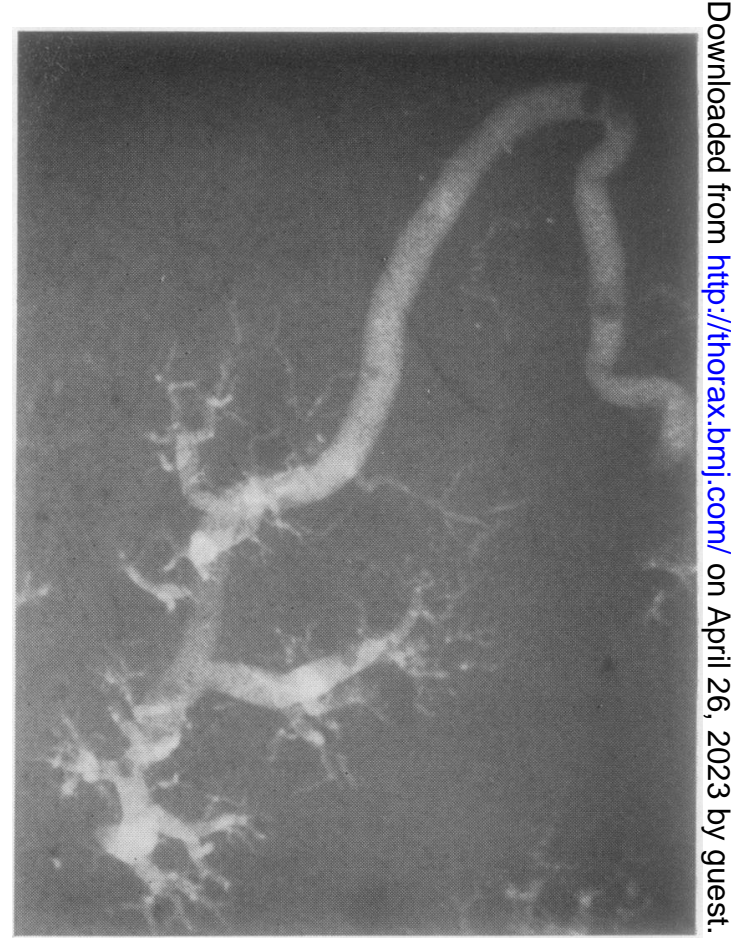

FIG. 2. Cor triatriatum. Systemic artery injection. $\frac{T}{0}$ An 'isolated' subpleural systemic-pulmonary end-to-end $\overrightarrow{\mathbb{D}}$ anastomosis is shown. The systemic origin of the pleural $\frac{\cap}{\mathbb{D}}$ branch was verified by tracing it back through the lung $\varrho$

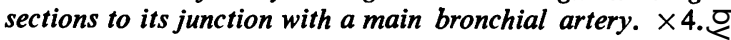



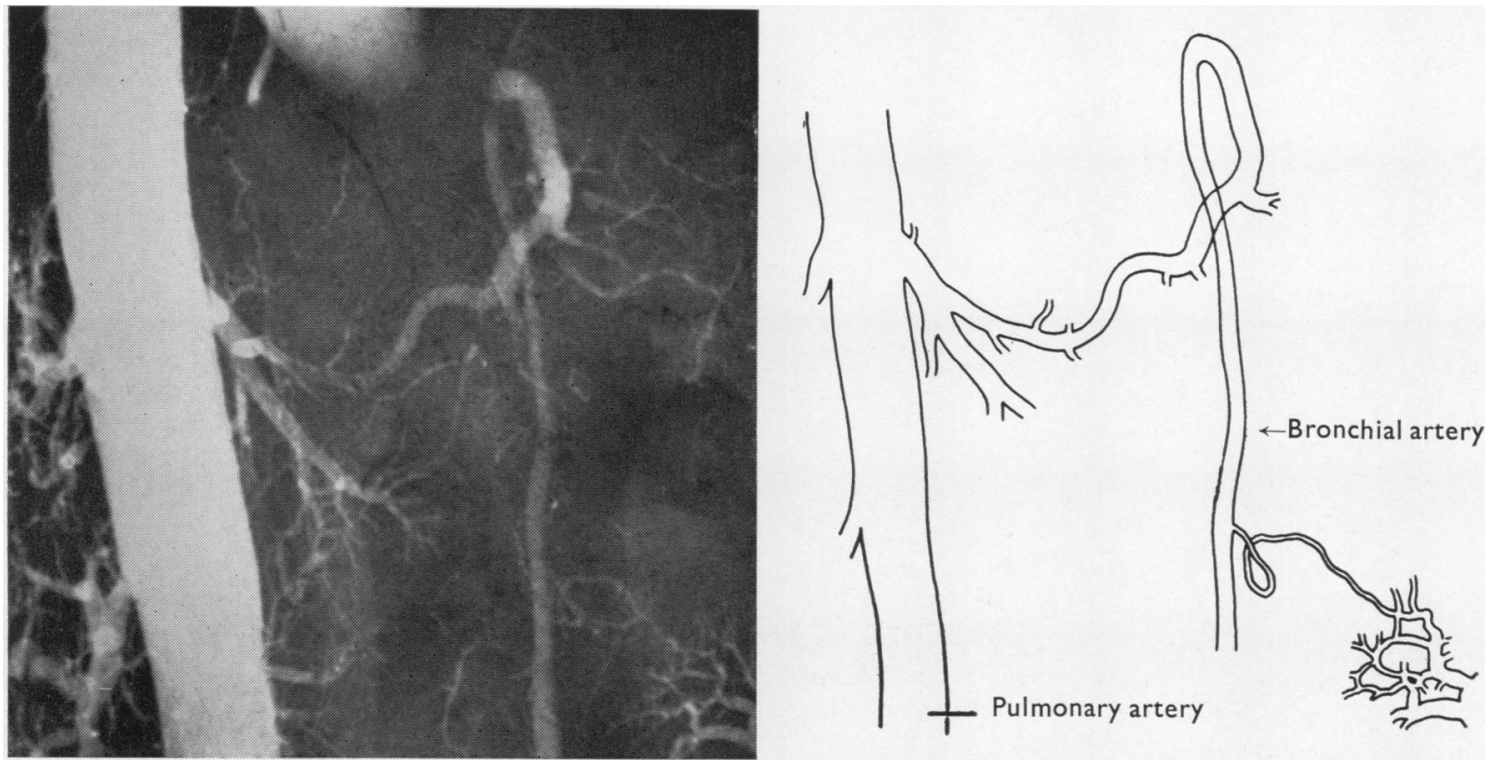

FIG. 3. Cor triatriatum. Systemic arterial injection. An 'isolated' bronchopulmonary anastomosis between a lateral branch from a fourth generation pulmonary artery and a bronchial artery lying on the wall of the accompanying bronchus. The end-to-end mode of communication is shown. It is probable that a minute peripheral anastomosis is shown in the lower right corner. $\times 5$.

in the lung was studied. In one lung where the systemic arteries only had been injected, no anastomoses were seen at the sites of 'angiomatoid' lesions, and this finding was confirmed by studying the pattern of the pulmonary artery injection to the opposite lung. The microradiographic appearance of the peripheral pulmonary arterioles at the sites of angiomatoid lesions was characteristic: the end of each pulmonary arteriole was surmounted by a terminal haze of injection medium where 'micropaque' had passed from the partially occluded pulmonary arteriole to the dilated thin-walled distal vessels (Fig. 4). Although injection material was shown to extend into the dilated vessels, no communication could be found between them and the systemic arterial bed.

The major abnormality of the systemic vascular supply to lungs with Eisenmenger's syndrome was related to extensive intravascular pulmonary artery thrombosis. Intense bronchial artery proliferation was found in the walls of the thrombosed pulmonary vessels where numerous systemic branches extended inwards through the media and intima to penetrate the intravascular blood clot (Fig. 5). The microradiographic appearance of this form of acquired systemic-pulmonary anastomosis was readily distinguished from the other forms previously described. Perivascular haemorrhage was found in many places in these lungs and was

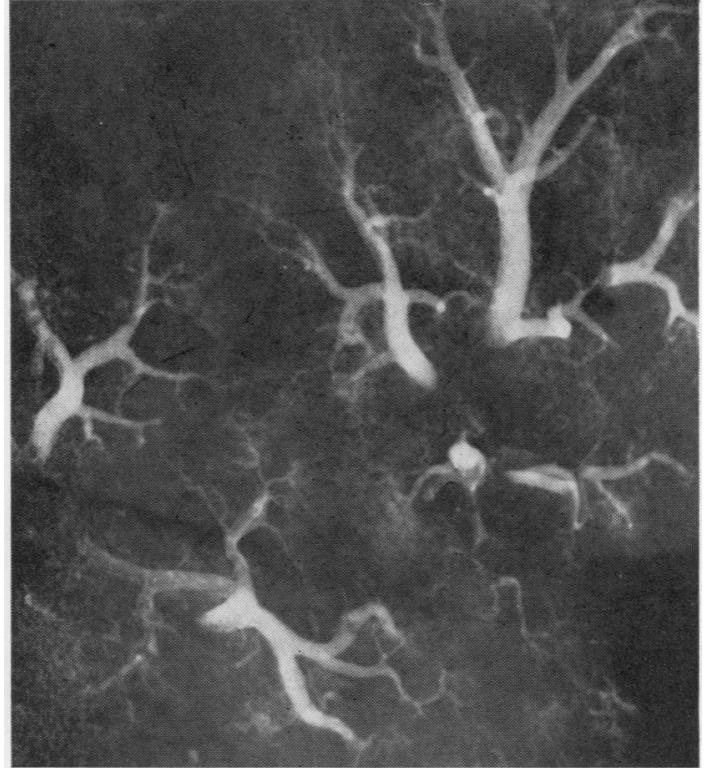

FIG. 4. Eisenmenger's complex. Pulmonary artery injection, showing the attenuated pattern of arterial branching with each terminal arteriole surmounted by $a$ 'haze' of injection medium. Microscopy showed that the appearance represented Micropaque which had extended into the dilated thin-walled pulmonary vessels distal to the partially occluded pulmonary arteriole. Note the absence of systemic arterial filling. $\times 5$. 

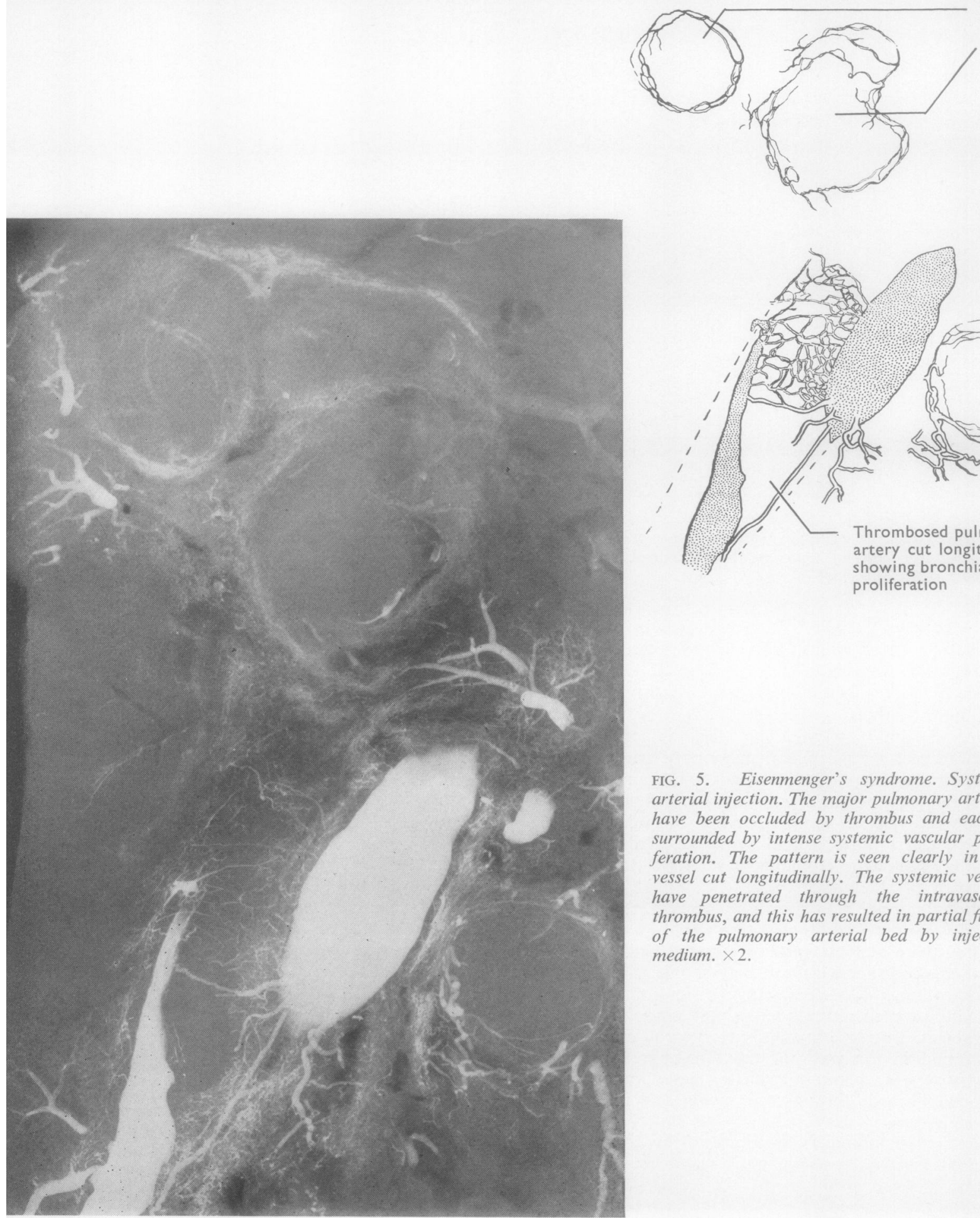

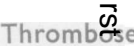
pulmongy arteries $\overline{0}$

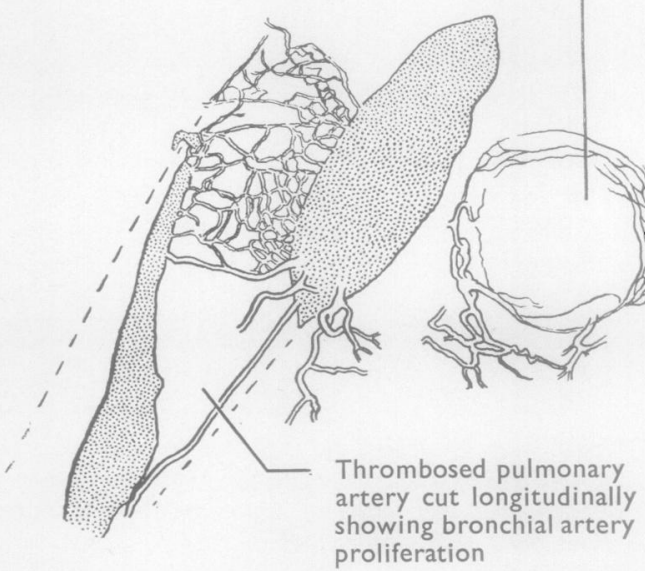

FIG. 5. Eisenmenger's syndrome. Systemic arterial injection. The major pulmonary arteries have been occluded by thrombus and each is surrounded by intense systemic vascular proliferation. The pattern is seen clearly in the vessel cut longitudinally. The systemic vessels have penetrated through the intravascular thrombus, and this has resulted in partial filling of the pulmonary arterial bed by injection medium. $\times 2$. 
always related to intense proliferation of the vasa vasorum in the neighbourhood of intravascular thrombosis.

The forms of anastomosis identified in these cases of congenital heart disease were compared with those found in some types of acquired lung disease.

ACQUIRED LUNG DISEASE WITH PULMONARY HYPERTENSION All lungs were examined for evidence of pulmonary hypertension. This was based on typical changes in the radiographic pattern of the small pulmonary artery branches as described by Short (1956), and on the increase of wall thickness compared with the lumen of these vessels studied in histological preparations (Heath and Edwards, 1958). This pathological evidence of pulmonary hypertension secondary to lung disease was found in 39 lungs. The underlying disease was diffuse pulmonary fibrosis (interstitial pulmonary fibrosis) in nine, scleroderma in two, sarcoidosis in two, emphysema and bronchitis in 18, and bronchiectasis in eight. In these lungs large 'isolated' subpleural systemic anastomoses were found irrespective of the nature of the underlying disease (Fig. 6). These connexions were similar in form

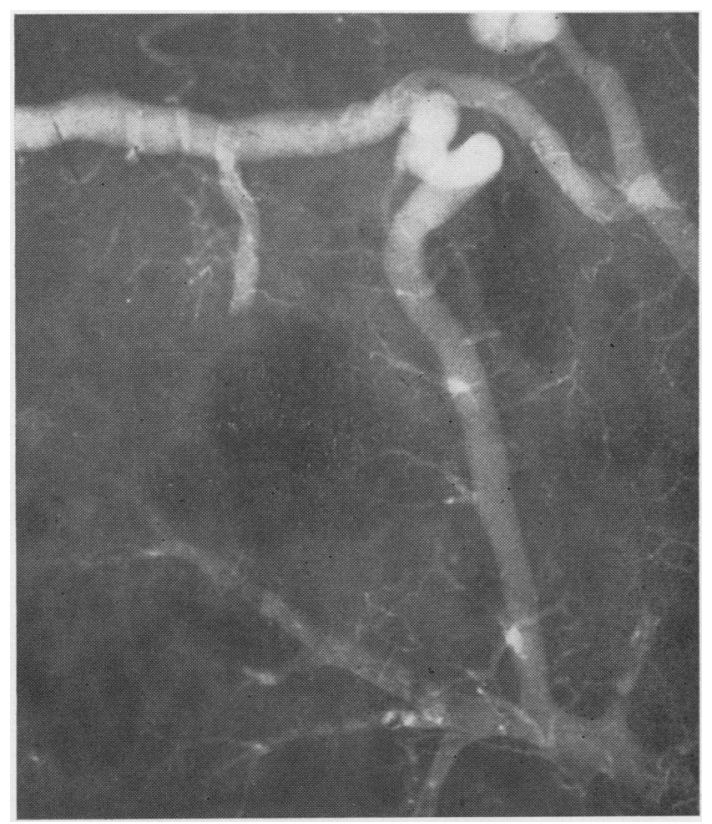

FIG. 6. Interstitial pulmonary fibrosis. Systemic artery injection showing an 'isolated' subpleural systemicpulmonary artery anastomosis. Abrupt change in the vessel wall structure occurred and confirmed the end-to-end mode of junction. $\times 11$. and distribution to those found in congenital heart disease and were quite distinct from those related to systemic vascular proliferation associated with inflammatory changes which will be described later. The largest subpleural systemic-pulmonary anastomosis found measured $1.5 \mathrm{~mm}$. in diameter, and longitudinal serial section demonstrated conclusively the end-to-end mode of junction. Fig. 7 shows the abrupt change in the vessel wall structure from the muscular media of the thickwalled systemic vessel to the well-defined internal and external elastic laminae of the pulmonary artery.

NEW TISSUE FORMATION WITHIN THE LUNG Quite distinct forms of anastomoses were found in relation to new tissue formation in the lungs, and the morphological pattern of these was found to be closely related to the underlying disease. For this reason the patterns seen in individual lung diseases will be summarized, although it is probable that, in all, the essential nature of the communication is dependent on the development of multiple connexions between minute pulmonary arterioles and proliferating branches of systemic vessels in areas of inflammatory or tumour tissue.

DIFFUSE PULMONARY FIBRosis Sixteen lungs were studied in which there was widespread fibrosis: interstitial pulmonary fibrosis in 10 lungs, scleroderma in two, sarcoidosis in two, and irradiation fibrosis in two lungs.

In 12 of the 16 lungs the bronchial vascular supply was greatly expanded to form an extensive network throughout the lungs. The highly vascular nature of the lesions in diffuse pulmonary fibrosis has been noted before (Golden and Bronk, 1953), but the origin of these vessels from the systemic circulation has not been demonstrated previously. In the neighbourhood of this vascular proliferation numerous fine systemic-pulmonary anastomoses have been found. Fig. 8 shows numerous branches from the systemic pleural vessels extending into the underlying lung and communicating with peripheral branches of the pulmonary artery. There are two reasons why the configuration of anastomoses in areas of vascular proliferation can be distinguished clearly from the 'isolated' communications described previously. Firstly, there are a large number of minute anastomoses found within a limited area of abnormal lung; and secondly, the pulmonary arterial component of the anastomosis is of small size and is no larger than the non-communicating pulmonary branches in the immediate neighbourhood (cf., Figs. 5, 6, and 7). A similar appearance was seen in irradiation 


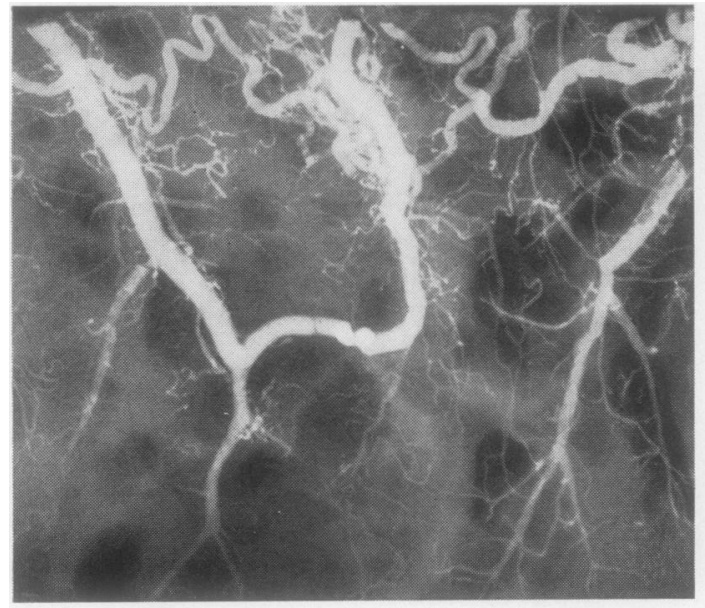

1
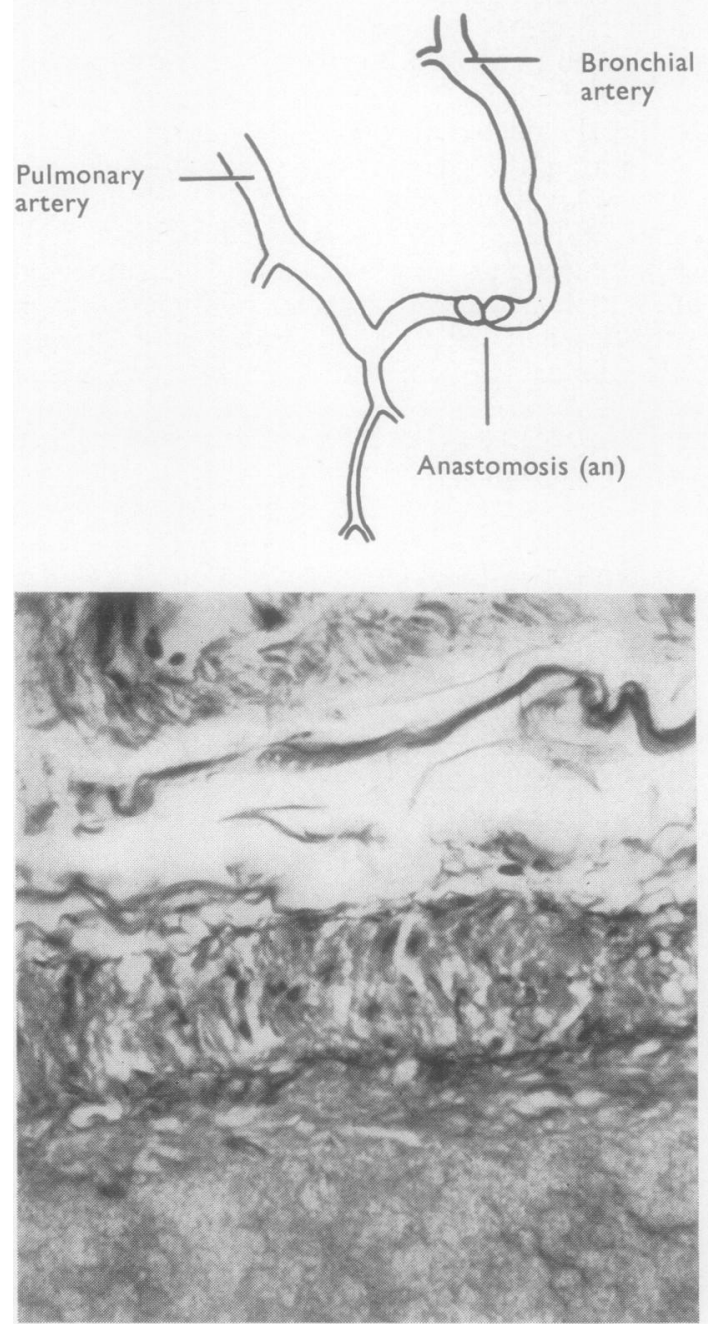

3

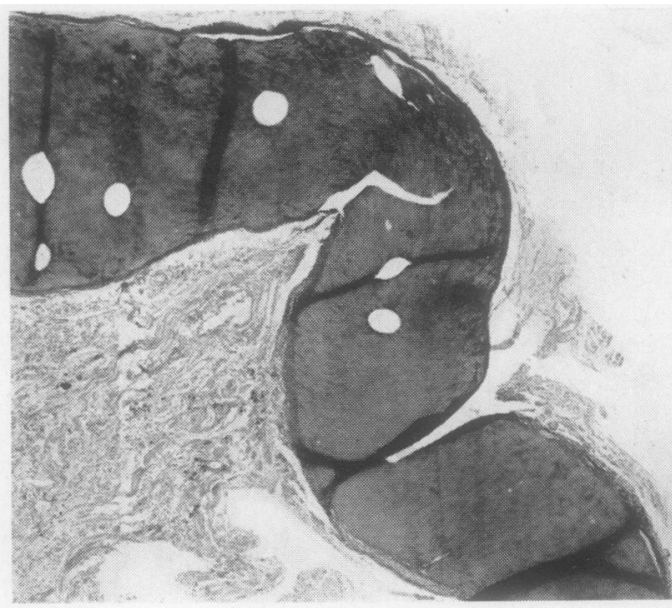

2
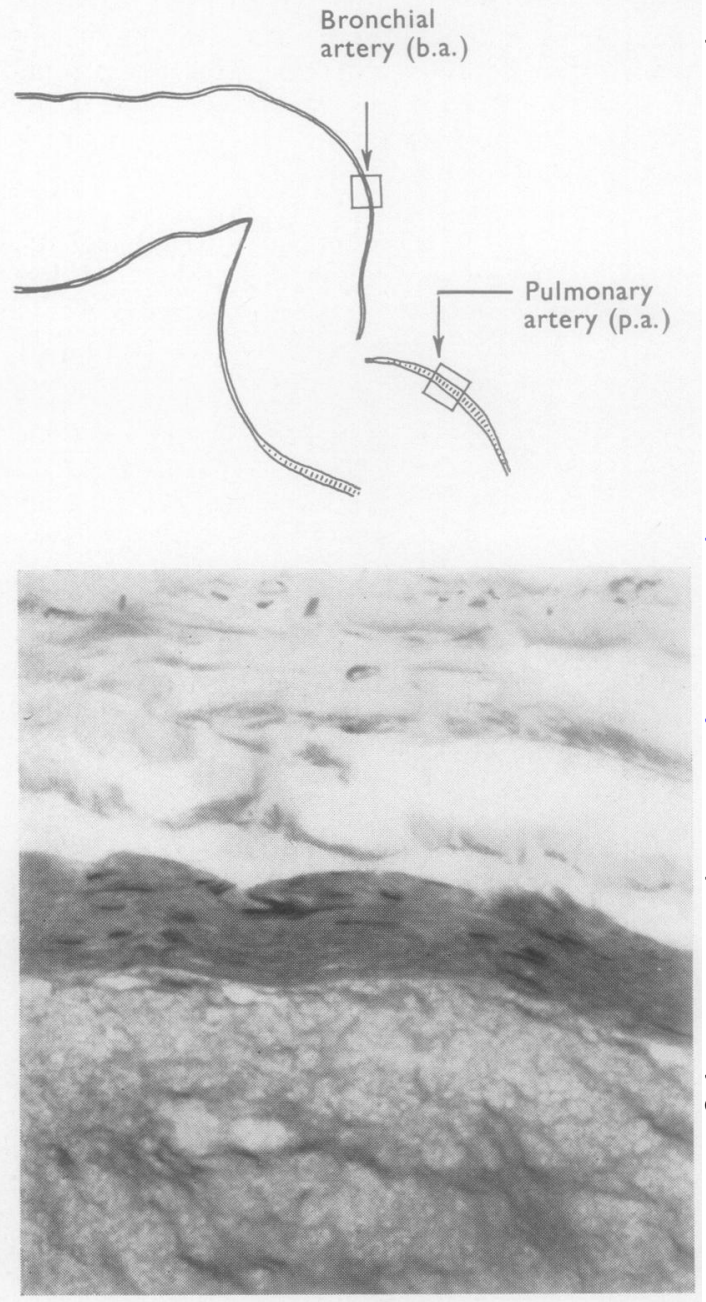

4

FIG. 7. Interstitial pulmonary fibrosis. An 'isolated' systemic-pulmonary anastomosis showing the end-to-end mode of junction. (1) Radiograph $\times 1 \cdot 5$. (2) Microscopic appearance of vessel marked (an) $\times 20$. (3) Vessel wall detail fromo the pulmonary artery end of the anastomosis (p.a.) $\times 400$. anastomosis (b.a.) $\times 400$.

(4) Vessel wall detail from the systemic artery end of the 

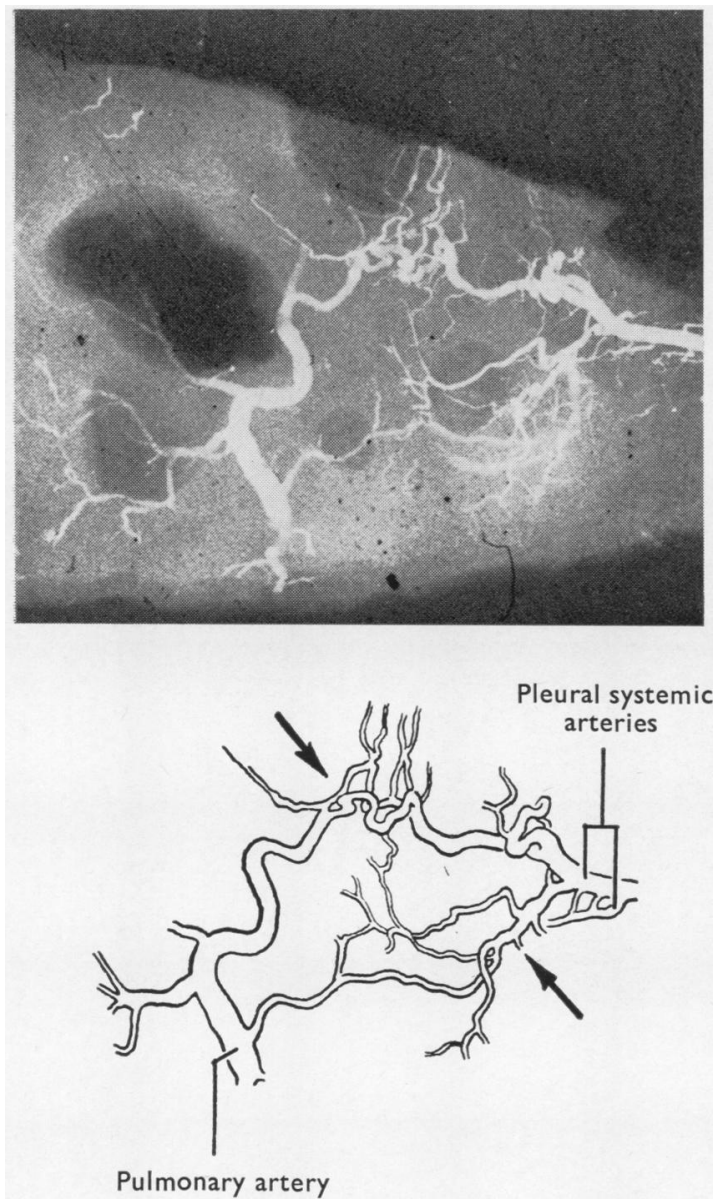

FIG. 8. Interstitial pulmonary fibrosis. Systemic artery injection showing proliferation of systemic pleural branches communicating at many points with minute branches of the pulmonary artery.

fibrosis where innumerable fine pleural vessels were found streaming into the underlying lung to communicate with the pulmonary vascular bed (Fig. 9). Serial sections confirmed that communication occurred between thin-walled arterioles of about $50 \mu$, which could be traced inwards to their junction with a muscular pulmonary arteriole, and outwards to the pleural surface to their point of continuity with a definitive systemic vessel. In these lungs no pleural adhesions were present, and the systemic vessels were derived from the bronchial arteries and not from intercostal vessels.

Within the lung, large numbers of minute anastomoses could be seen in relation to the expanded systemic vascular bed. The bronchial artery shown in Fig. 10 accompanied a fourth generation bronchus and gave lateral branches which communicated with a distant pulmonary artery at several points. A similar form of anastomosis was found in scleroderma lungs (Fig. 11): here fine branches of the pulmonary artery have been filled through the proliferating systemic vascular network.

BRONCHIECTASIS In bronchiectatic lungs two forms of anastomoses were found: one in relation to systemic vessel proliferation in the walls of bronchiectatic sacs and areas of destroyed lung, and the other proximally along axial pulmonary arteries. The latter form has been described previously by Liebow et al. (1949) and by Cockett and Vass (1951) and appears to be a characteristic vascular feature in bronchiectasis but was not seen in any other lung disease. These proximal anastomoses were found between parallel lying bronchial and axial pulmonary arteries, the two being linked in a side-to-side manner by a connecting vessel with a distinct wall of circular muscle (Fig. 12). The number of anastomoses of this type found within single lobes was usually less than six, and although they have been seen at several points between the third and sixth generation bronchi along the same axial pulmonary artery, the most common site was at the termination of the axial pulmonary vessel. The origin of this form of anastomosis will be discussed later.

TUMOUR TISSUE The vascular supply to primary carcinoma of the lung is derived from the systemic circulation (Wright, 1938 ; Wood and Miller, 1938 ; Cudkowicz and Armstrong, 1953b ; TurnerWarwick, 1961).

Filling of the pulmonary artery following bronchial artery injection was found in nine out of 21 lungs with primary bronchial carcinoma. The sites of these anastomoses were often widespread; they were found in the carcinomatous lobe in seven out of 21 lungs, in the non-carcinomatous lobe on the same side in eight out of 21 , and in the opposite lung in five instances. However, emphysema or bronchiectasis was also present in the majority of these lungs and it is uncertain what part the tumour played in the development of anastomoses. This has been discussed in detail elsewhere (Turner-Warwick, 1961).

THROMBOSIS OF MAJOR PULMONARY ARTERIES Systemic-pulmonary anastomoses found in relation to pulmonary artery thrombosis and acquired lung disease have quite a distinct microradiographic pattern. This is identical with that described in 


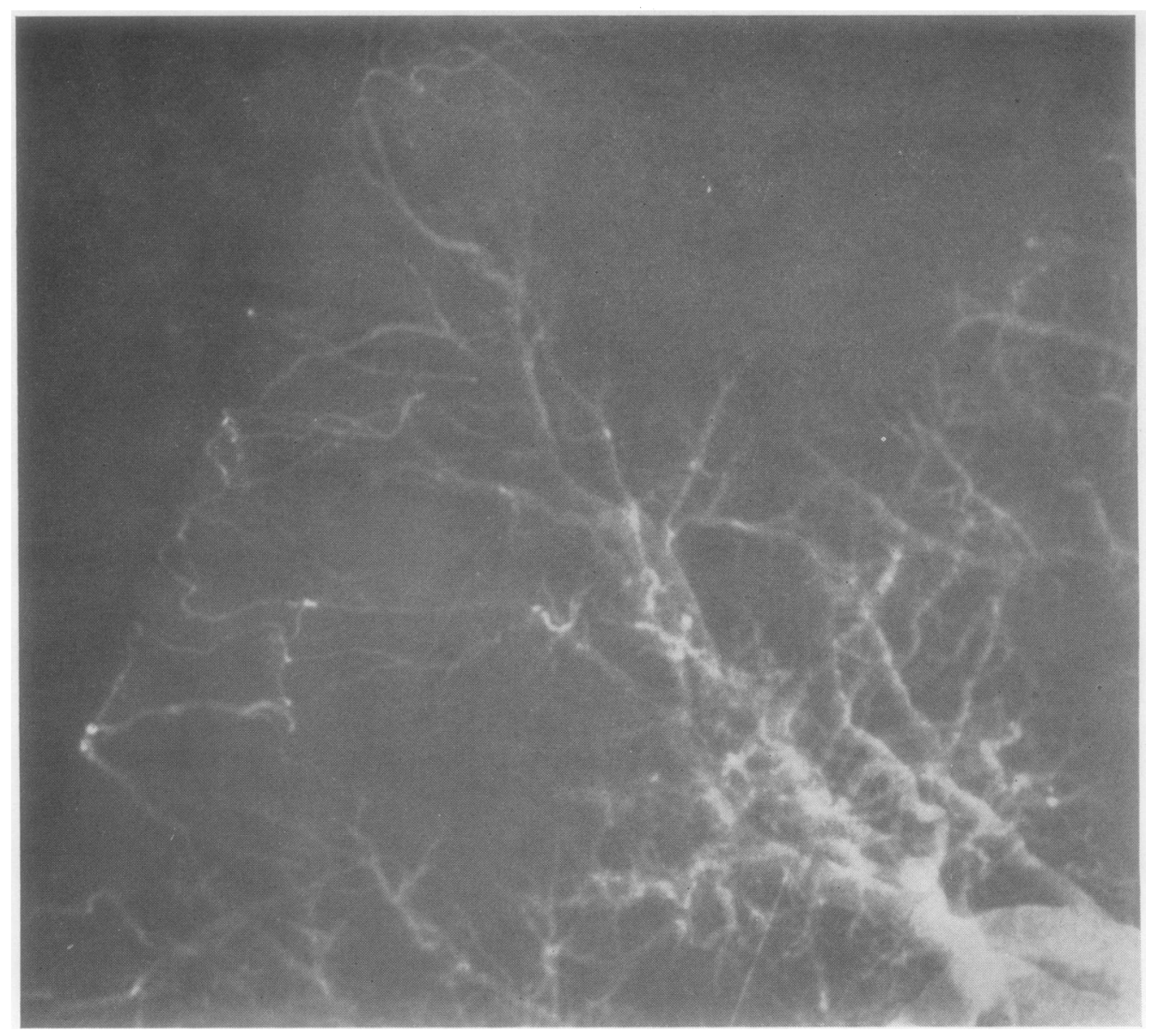

FIG. 9. Irradiation pneumonitis with fibrosis. Proliferation of the pleural systemic vessels and their communication at many points with minute pulmonary artery branches. The systemic arteries only were injected, and the pulmonary artery filling has occurred through anastomoses.
Pleural systemic arteries

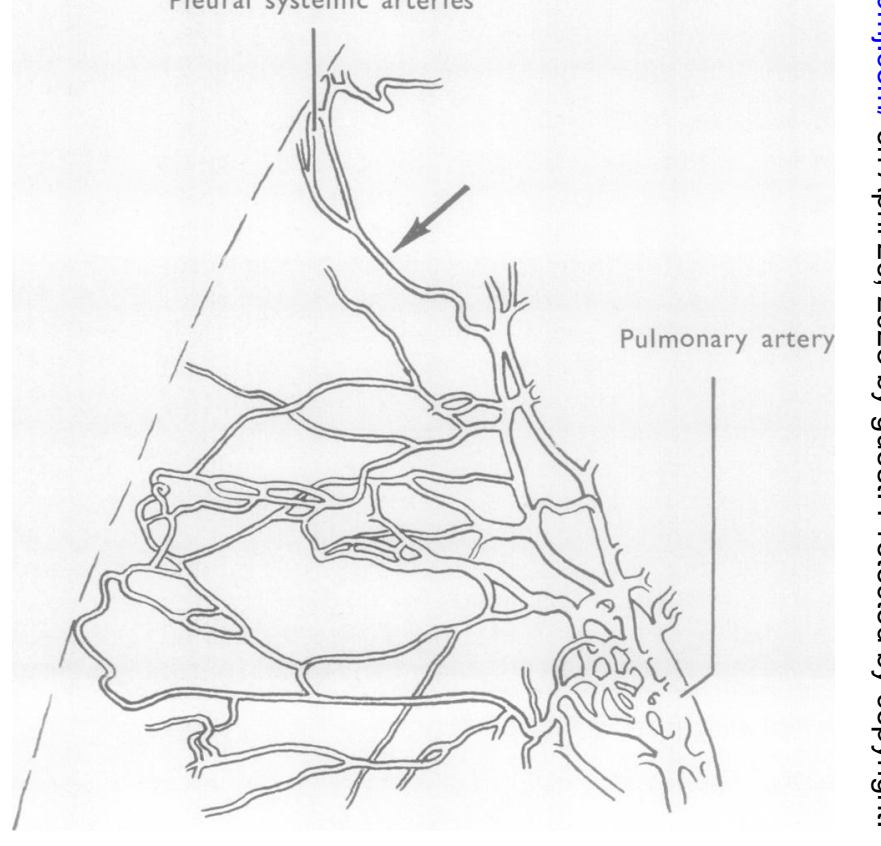




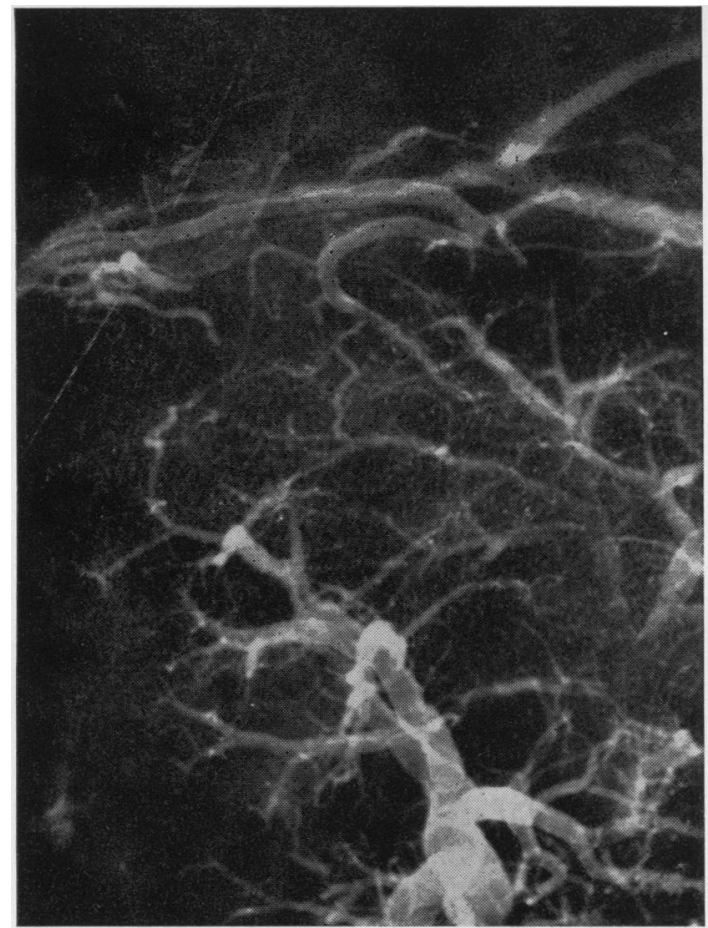

FIG. 10

Bronchial arteries

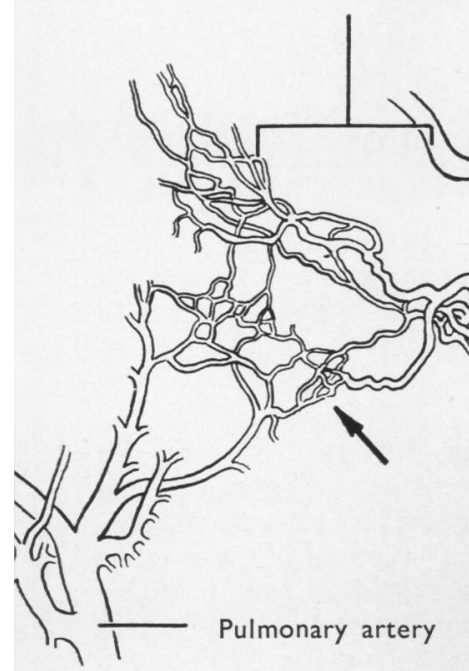

Bronchial artery

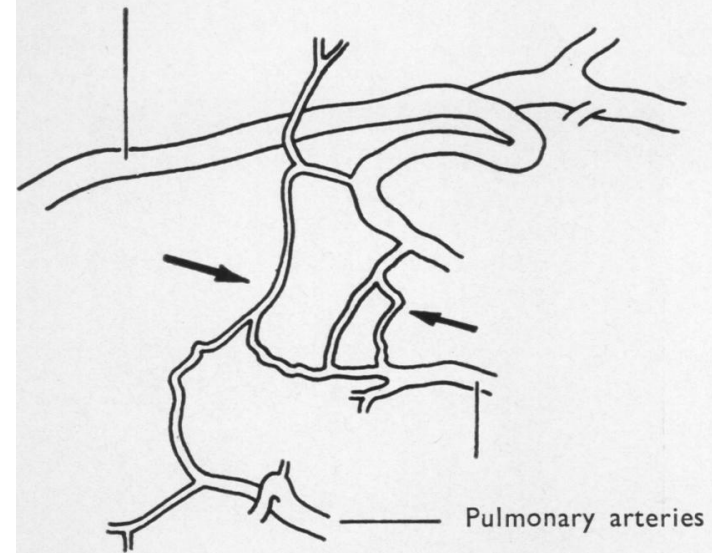

FIG. 10. Interstitial pulmonary fibrosis. Systemic arterial injection. Intrapulmonary systemic-pulmonary anastomoses between a bronchial artery branch accompanying a fourth generation bronchus and minute pulmonary artery branches arising from a neighbouring axial system. $\times 5$.

FIG. 11. Sclerederma. Systemic-pulmonary communication in an area of bronchial artery proliferation. The systemic arteries only were injected and the pulmonary artery filling has occurred through anastomosis. $\times 5$.

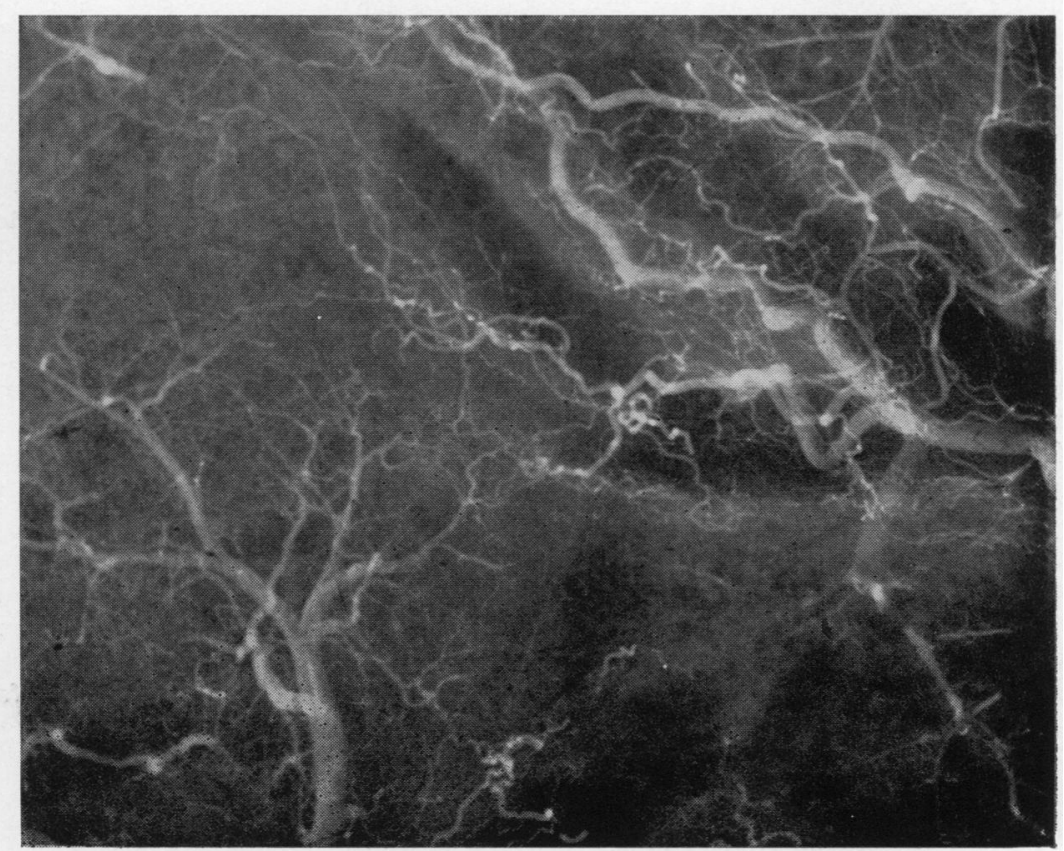



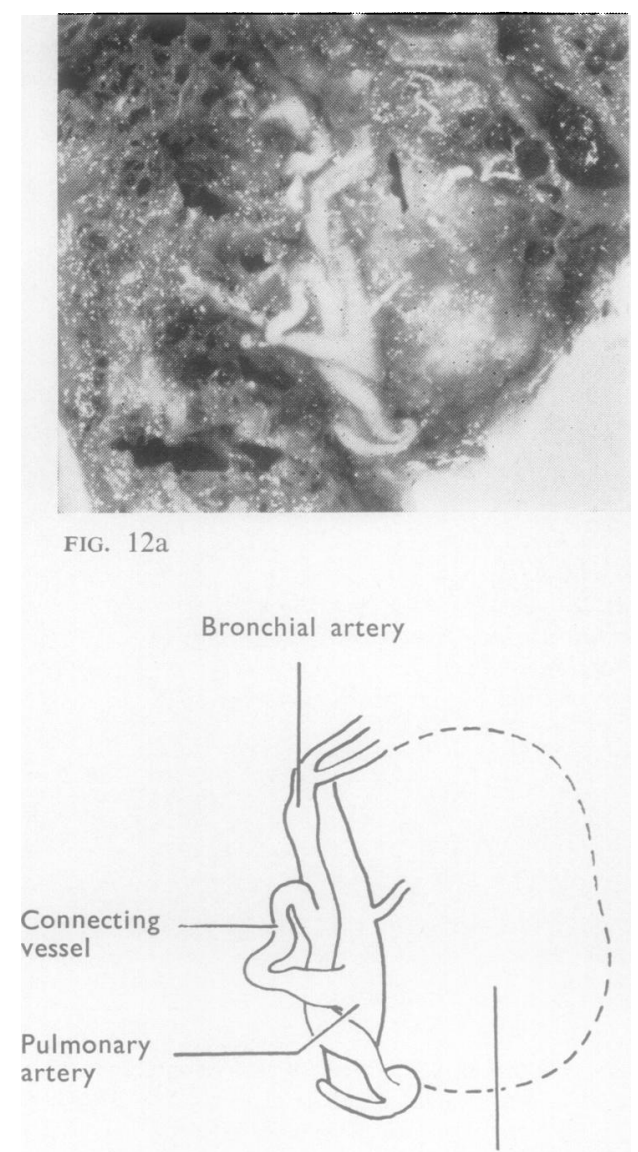

Bronchiectatic sac the lungs with Eisenmenger's syndrome (Fig. 5) and bears no resemblance to any of the other forms of end-to-end connexions described above.

\section{DISCUSSION}

By comparing the morphology of anastomoses occurring in congenital heart disease, where the lungs are otherwise normal, with that found in acquired lung disease, it is possible to suggest which morphological types of anastomoses are likely to be due to enlargement of pre-existing channels and which may be acquired and newly developed.

The large end-to-end anastomoses between systemic pleural arteries and the pulmonary artery were found not only in many congenital heart conditions (Fallot's tetralogy, cor triatriatum, and Eisenmenger's ventricular septal defect) but also

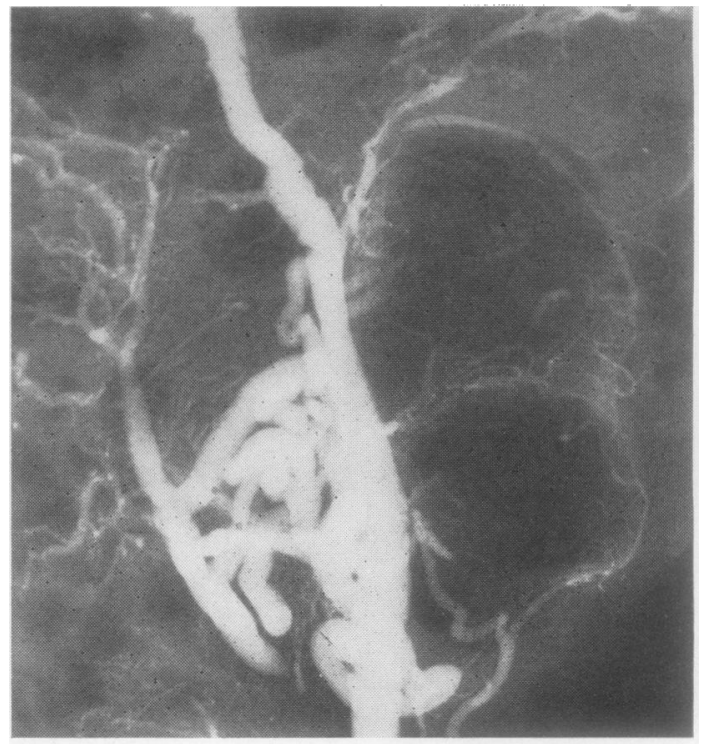

FIG. $12 b$

FIG. 12. (a) Bronchiectasis. Systemic artery injection. Photograph of a proximal bronchopulmonary anastomosis; the axial pulmonary artery is linked to the bronchial artery by a distinct communicating vessel. $\times 2$.

(b) Bronchiectasis. Radiograph of the anastomosis illus trated in Fig. 12a. Fine communications between the bronchial and pulmonary artery branches are also seen on the wall of a bronchiectatic sac. $\times 6$.

in a wide variety of lung diseases, especially in cases with acquired pulmonary hypertension. It is suggested that these 'isolated' subpleural systemicpulmonary anastomoses represent pre-existing channels which have increased in size, probably in relation to the altered haemodynamics within the pulmonary arterial bed. Numerous end-to-end systemic-pulmonary anastomoses have been found $\sigma$ within the lung in cases with congenital heart $N$ disease; these have a constant distribution and N form and have been described in detail. Their morphology differs in some respects from the peripheral end-to-side connexions described by Hales and Liebow (1948) and is quite dissimilar to the proximal end-to-side anastomoses described by him. In the present work large tortuous bronchial arteries were seen closely applied to the first few generations of the axial pulmonary arteries but direct continuity between the two sets of vessels was never found. 
The 'isolated' anastomoses seen in congenital heart disease and some acquired lung diseases contrast sharply with the dense networks of endto-end arteriolar anastomoses between the pulmonary and the greatly expanded systemic arterial circulations in the neighbourhood of inflammatory tissue. This type of communication has not been seen in congenital heart disease and these channels are thought to represent newly developed anastomotic pathways, as suggested earlier by Liebow et al. (1949).

The origin of the proximal anastomoses seen in bronchiectasis is uncertain. Their proximal position suggests that they may represent 'isolated' intrapulmonary anastomoses, greatly distorted by the destruction of lung tissue and obliteration of the peripheral pulmonary arterial bed. Harris and Heath (1962) have suggested a similar mode of formation for these anastomoses.

Systemic-pulmonary anastomoses at the site of pulmonary artery thrombosis are acquired and clearly distinct from the various other end-to-end forms described. The latter have been found where there is no microscopic evidence of pulmonary artery obstruction.

The suggestion made by Cudkowicz and Armstrong (1953a) that the formation of anastomoses in acquired lung disease depended on obstruction and thrombosis of the pulmonary arterial bed is not upheld. Likewise the suggestion that thrombosis plays an important role in the development of anastomoses in Fallot's tetralogy (Hales and Liebow, 1948) is not supported.

\section{SUMMARY}

The morphology of systemic-pulmonary anastomoses in acquired lung disease has been contrasted with that found in the lungs from patients with some forms of congenital heart disease.

Several forms of anastomoses have been identified, some of which have not been described in detail before.

Morphological evidence suggests that certain forms of end-to-end anastomosis found in both acquired lung and congenital heart disease represent enlargement of pre-existing channels. Other distinct forms have been described which were seen only in relation to inflammatory or tumour tissue, and these probably represent newly formed communications.

I wish to thank all the physicians and surgeons at the Brompton Hospital who generously allowed me to study their cases. I am especially grateful to Dr. Clifford Hoyle and Professor J. G. Scadding for their encouragement and to Professor Liebow of Yale University for his help and kindness to me.

I should like to thank Mr. Alan Curd who prepared the photographs, Mr. Drury for the illustrations and Miss Doreen Coombs for serial sections. Ilford's Ltd. kindly developed the 'Line' radiographs.

Part of this work was done while I was in receipt of a research grant from the Brompton Hospital Research Committee ; I should like to thank them for this and for a travel grant. I have pleasure in thanking the Wellcome Foundation for a travel fellowship to visit the United States in the course of this work.

\section{REFERENCES}

Cockett, F. B., and Vass, C. C. N. (1951). A comparison of the role of the bronchial arteries in bronchiectasis and in experimental ligation of the pulmonary artery. Thorax, 6, 268.

Cudkowicz, L., and Armstrong, J. B. (1951). Observations on the normal anatomy of the bronchial arteries. Ibid., 6, 343.

- (1953a). The bronchial arteries in pulmonary emphysema. Ibid. 8,46 .

- - (1953b). The blood supply of malignant pulmonary neoplasms. Ibid., 8, 152.

Golden, A., and Bronk, T. T. (1953). Diffuse interstitial fibrosis of lungs. Arch. intern. Med., 92, 606.

Hales, M. R., and Liebow, A. A. (1948). Collateral circulation to the lungs in congenital pulmonic stenosis. Bull. int. Ass. med. Mus., $28,1$.

Harris, P., and Heath, D. (1962). The Human Pulmonary Circulation. Its Form and Funct ion in Health and Disease. Livingstone, London. Heath, D., and Edwards, J. E. (1958). The pathology of hypertensive pulmonary vascular disease. Circulation, 18, 533 .

Liebow, A. A., Hales, M. R., and Lindskog, G. E. (1949). Enlargement of the bronchial arteries, and their anastomoses with the pulmonary arteries in bronchiectasis. Amer. J. Path., 25, 211. Bloomer, W. E., Harrison, W., and Lindskog, G. E. (1950). Studies on the lung after ligation of the pulmonary artery. II. Anatomical changes. Ibid., 26, 177.

- - (1959). In Pulmonary Circulation. An International Symposium, 1958, ed. W. R. Adams and I. Veith. Grune and Stratton, New York. Chapter beginning on p. 79. 'Relation of Bronchial to Pulmonary Vascular Tree'.

Miller, W. S. (1947). The Lung, 2nd ed. Thomas, Springfield, Illinois Short, D. S. (1956). Post-mortem pulmonary arteriography, with special reference to the study of pulmonary hypertension. J. Fac. Radiol. (Lond.), 8, 118

Turner-Warwick, M. (1961). The bronchial artery patterns in pulmonary and cardiovascular disease. Ph.D. Thesis, London University.

Verloop, M. C. (1948). The bronchial arteries and their anastomoses with the arteriae pulmonales in the human lung: a microanatomical study. Acta anat. (Basel), 5, 171 .

Weibel, E. (1959). Die Blutgefässanastomosen in der menschlichen lunge. $Z$. Zellforsch., 50,653 .

Wood, D. A., and Miller, M. (1938). The role of the dual pulmonary circulation in various pathologic conditions of the lungs. $J$. thorac. Surg., 7, 649 .

Wright, R. D. (1938). The blood supply of abnormal tissues in the lungs. J. Path. Bact., 47, 489. 